\title{
The Thermal Properties of Pseudoscalar Heavy Quarkonium
}

\author{
E. Veli Veliev* \\ Department of Physics, Kocaeli University, 41380 Izmit, Turkey \\ E-mail: Elsendkocaeli.edu.tr
}

\section{K. Azizi}

Physics Division, Faculty of Arts and Sciences, Doğuş University, Acıbadem-Kadıköy, 34722 Istanbul, Turkey

E-mail: kaziziedoqus.edu.tr

\section{H. Sundu}

Department of Physics, Kocaeli University, 41380 Izmit, Turkey

E-mail: havrive.sundudkocaeli.edu.tr

\section{N. Akşit}

Faculty of Education, Kocaeli University, 41380 Izmit, Turkey

E-mail: nurcanaksitokecaeli.edu.tr

\begin{abstract}
Study of heavy quarkonia is one of the most interesting fields and plays an important role in understanding the properties of QCD vacuum as well as nonperturbative behavior of strong interaction. In this study we investigated the temperature dependence of leptonic decay constants for pseudoscalar heavy quarkonium states. We took into account the additional operators in the Wilson expansion at finite temperature and calculated thermal spectral density in the QCD side. The analysis of obtained thermal QCD sum rules shows that at critical temperature the decay constant decreases approximately $50 \%$ for $\eta_{c}$ and $57 \%$ for $\eta_{b}$ and this situation can be seen of a sign of QGP phase transition. The results at zero temperature are in a good consistency with the existing experimental values as well as predictions of the other nonperturbative approaches.
\end{abstract}

The 2011 Europhysics Conference on High Energy Physics-HEP 2011,

July 21-27, 2011

Grenoble, Rhône-Alpes France

\footnotetext{
* Speaker.
} 


\section{Temperature Behavior of the Decay Constants}

The discussion of heavy-meson properties has a rather long history and the heavy mesons play a very important role in our understanding of nonperturbative dynamics of QCD. Recently, an important progress has been achieved by BABAR Collaboration and they observed $\eta_{b}$ in the photon spectrum of $\Upsilon(3 S) \rightarrow \gamma \eta_{b}$ [四]. The interpretation of in-medium hot matter effects requires investigation of hadron properties at finite temperature. Our aim in this work is to investigate the temperature dependence of leptonic decay constants of the pseudoscalar $\eta_{b}$ and $\eta_{c}$ mesons. Using the thermal quark propagator, we find the imaginary part of the correlation function in the following form:

$$
\operatorname{Im} \Pi\left(q_{0}, T\right)=-q_{0} N_{c} \int \frac{d \mathbf{k}}{8 \pi^{2} \omega}\left(1-2 n(\omega)+n^{2}(\omega)\right) \delta\left(q_{0}-2 \omega\right),
$$

To calculate the nonperturbative part in QCD side, we use the nonperturbative part of the quark propagator in an external gluon field, $A_{\mu}^{a}(x)$ in the Fock-Schwinger gauge, $x^{\mu} A_{\mu}^{a}(x)=0$. Taking into account one and two gluon lines attached to the quark line, the massive quark propagator up to terms necessary for our calculations can be written as:

$$
\begin{aligned}
S^{a a^{\prime} \text { nonpert }}(k) & =-\frac{i}{4} g\left(t^{c}\right)^{a a^{\prime}} G_{\kappa \lambda}^{c} \frac{1}{\left(k^{2}-m^{2}\right)^{2}}\left[\sigma_{\kappa \lambda}(\not k+m)+(\not k+m) \sigma_{\kappa \lambda}\right] \\
& +\frac{i g^{2} \delta^{a a^{\prime}}}{9\left(k^{2}-m^{2}\right)^{4}}\left\{\frac{3 m\left(k^{2}+m \not k\right)}{4}\left\langle G_{\alpha \beta}^{c} G^{c \alpha \beta}\right\rangle+\left[m\left(k^{2}-4(k \cdot u)^{2}\right)\right.\right. \\
& \left.\left.+\left(m^{2}-4(k \cdot u)^{2}\right) \not k+4(k \cdot u)\left(k^{2}-m^{2}\right) \not h\right]\left\langle u^{\alpha} \Theta_{\alpha \beta}^{g} u^{\beta}\right\rangle\right\} .
\end{aligned}
$$

where $u^{\mu}$ is the four-velocity of the heat bath and $\Theta_{\lambda \sigma}^{g}$ is the traceless, gluonic part of the energymomentum tensor of the QCD. Taking into account additional operators arising at finite temperature, nonperturbative part of the correlation function can be expressed as:

$$
\begin{aligned}
& \hat{B} \Pi^{n p}=\int_{0}^{1} d x \frac{1}{24 \pi M^{6} x^{4}(-1+x)^{4}} \exp \left[\frac{m^{2}}{M^{2} x(-1+x)}\right]\left\{\langle \alpha _ { s } G ^ { 2 } \rangle \left[m^{6}(1-2 x)^{2}\right.\right. \\
& \left.+6 M^{2} m^{2} x^{2}(-1+x)^{2}-12 M^{6} x^{4}(-1+x)^{4}+6 M^{4} m^{2} x^{2}(-1+x)^{2}\left(-1-4 x+4 x^{2}\right)\right] \\
& +\alpha_{s}\left\langle\Theta^{g}\right\rangle\left[\left[\left(m^{6}(1-2 x)^{2}+M^{6} x^{3}(-1+x)^{3}\left(9-11 x+11 x^{2}\right)-m^{2} M^{4}(-1+x)^{2} x^{2}\right.\right.\right. \\
& \left.\left.\left.\left.\times\left(1-17 x+17 x^{2}\right)-2 m^{4} M^{2} x\left(-1+2 x-2 x^{2}+x^{3}\right)\right)\right]\right]\right\},
\end{aligned}
$$

where $M^{2}$ is the Borel mass parameter and $\omega=\sqrt{\mathbf{k}^{2}+m^{2}}$. Matching the phenomenological and QCD sides of the correlation function, sum rules for the decay constant of pseudoscalar meson is obtained:

$$
f_{P}^{2}(T) m_{P}^{4}(T) \exp \left(-\frac{m_{P}^{2}}{M^{2}}\right)=4 m^{2}\left\{\int_{4 m^{2}}^{s_{0}(T)} d s\left(\rho^{a, p e r t}(s)+\rho_{\alpha_{s}}(s)\right) \exp \left(-\frac{s}{M^{2}}\right)+\widehat{B} \Pi^{n p}\right\},
$$

where $\rho^{a, p e r t}(s)$ and $\rho_{\alpha_{s}}(s)$ are annihilation part of thermal spectral density and the perturbative two-loop order correction, respectively. 


\section{Conclusions and Discussions}

The sum rules for the masses and decay constants include two auxiliary parameters, namely continuum threshold $s_{0}$ and Borel mass parameter $M^{2}$. Therefore, we should look for working regions for these parameters such that the dependences of the masses and decay constants on these parameters is weak. The continuum threshold, $s_{0}$ is related to the energy of the first exited state with the same quantum numbers as the interpolating currents. We choose the values $11 \mathrm{GeV}^{2} \leq$ $s_{0} \leq 12 \mathrm{GeV}^{2}$ and $97 \mathrm{GeV}^{2} \leq s_{0} \leq 100 \mathrm{GeV}^{2}$ for the continuum threshold in accordance with $\eta_{c}$ and $\eta_{b}$ channels, respectively.

Taking into account the temperature dependencies of hadronic threshold, energy density, quark and gluon condensates, we obtained that decay constants are well described by the following fit functions:

$$
\begin{gathered}
f_{\eta_{b}}(T)=-2.09497 \times 10^{-4} \exp (44.3066 T)+0.69914 \\
f_{\eta_{c}}(T)=-4.41028 \times 10^{-5} \exp (49.776 T)+0.42098 .
\end{gathered}
$$

Here decay constants and temperature are expressed in units of $\mathrm{GeV}$. These parameterizations are valid only in the interval $0 \leq T \leq 0.17 \mathrm{GeV}$.

Our investigations show that the masses and decay constants remain unchanged approximately up to $T \simeq 100 \mathrm{MeV}$, but after this point, they start to diminish with increasing the temperature. Near the critical or deconfinement temperature, the decay constants reach approximately to $50 \%, 57 \%$ of their values in vacuum, while the masses are decreased about $12 \%, 2 \%$ comparing with their values at zero temperature for $\eta_{c}, \eta_{b}$ mesons, respectively.

At $T=0$, values of the masses and leptonic decay constants for $\eta_{c}$ and $\eta_{b}$ are obtained as $m_{\eta_{c}}=2.996 \pm 0.035 \mathrm{GeV}, f_{\eta_{c}}=0.420 \pm 0.015 \mathrm{GeV}, m_{\eta_{b}}=9.650 \pm 0.034 \mathrm{GeV}$ and $f_{\eta_{b}}=0.696 \pm$ $0.049 \mathrm{GeV}$. The obtained results are in good consistency with the existing experimental data and predictions of other nonperturbative models [] (for more details see []]). Our results on the leptonic decay constants of $\eta_{b}$ and $\eta_{c}$ at zero temperature as well as the behavior of the masses and decay constants of the considered pseudoscalar heavy mesons with respect to the temperature can be checked in the future experiments.

\section{Acknowledgments}

This work has been supported in part by the Scientific and Technological Research Council of Turkey (TUBITAK) under the project no. 110T284 and Research Fund of Kocaeli University under grant no.2011/029.

\section{References}

[1] B. Aubert, et.al, BABAR Collaboration Phys. Rev. Lett. 101, 071801 (2008).

[2] K. W. Edwards et.al, CLEO Collaboration, Phys. Rev. Lett. 86, 30 (2001).

[3] E. Veli Veliev, K. Azizi, H. Sundu, N. Akşit, J. Phys. G: Nucl. Part. Phys. 39 (2012) 015002. 